\title{
Osteossínteses de ílio e fêmur em cachorro-do-mato (Cerdocyon thous)
}

\author{
Ilium and Femur Osteosynthesis in Crab-Eating Fox (Cerdocyon thous) \\ Bruna Zafalon da Silva', Eduardo Almeida Ruivo dos Santos' ${ }^{1}$ Priscila Medina da Costa ${ }^{2}$, \\ Miúriel de Aquino Goulart', Bernardo Schmitt' ${ }^{1}$ \& Marcelo Meller Alievi ${ }^{1,2}$
}

\begin{abstract}
Background: Fractures and dislocations are common orthopedic conditions arising from traumas from anthropic interaction on wild species, such as those caused by road trampling. Among the treatments, osteosynthesis is considered the method of choice in the treatment of fractures, such as ilium body fractures and femoral fractures. Based on this context, with regard to the incidence of auto accidents and few studies describing osteosynthesis techniques for crab-eating fox, the present study aimed to report the osteosynthesis performed on the body of the ileum and femur of a wild specimen of Cerdocyon thous. Case: A female, adult, crab-eating fox (Cerdocyon thous), coming from wild life was referred to hospital care with a history of run over. After specific examinations, it was diagnosed a complete transverse femoral shaft fracture, an oblique ilium body fracture, a pubic and ischial fracture, and pelvic canal narrowing. The treatment instituted was osteosynthesis of the femoral and ilium body fractures through the use of a $2.7 \mathrm{~mm}$ locking plate and screws. In the postoperative period, radiographic examination was performed, where bone callus formation was observed in the 5th week and bone remodeling and consolidation in the 11th week. After 140 days of rehabilitation, the animal was able to be released in the wild, with effective return of function of the pelvic limbs. The release was performed by environmental agencies in a national natural reserve, using radio necklace monitoring.

Discussion: Osteosynthesis techniques and preoperative and postoperative management proved to be effective for the return of adequate function of the pelvic limbs in Cerdocyon thous. For wild animals, the use of internal orthopedic implants (plate and screws, for example) become the most suitable for osteosynthesis. They decrease the risks of complications resulting from the management, since they require minimal postoperative manipulation. Thus, they maintain the wild behavior of the animal even in captivity. Among the complications observed in the clinical case, we can mention the use of long plate in the osteosynthesis of the ilium body and the narrowing of the pelvic canal as the most relevant. In relation to the pelvic canal narrowing and the reproductive cycle of the species, the crab-eating fox presents monogamous behavior, gestation of 3 to 6 pups per litter and breastfeeding for approximately 3 months. Because litters are relatively large (number of individuals per calving), the pups are relatively small - approximately $120 \mathrm{~g}$ at birth -, so it is expected that no complications occur during the gestational period. The treatment of fractures with the use of locking plates was efficient, obtaining bone consolidation at 10 weeks postoperatively, thus corroborating the scientific descriptions for bone consolidation in canids. Finally, the destination for release and monitoring by radiotelemetry was a joint decision between the environmental agencies of the State and the Union. The release decision was based on the maintenance of the wild behavior without evidence of meekness of the specimen, the absence of sanitary restrictions, as well as the population biology and the genetic flow since the release of the animal was carried out near the rescue site. It is concluded that the surgical treatment instituted by the femoral and ilium osteosynthesis was effective for the Cerdocyon thous specimen, culminating in the rehabilitation of the animal and release in the wild.
\end{abstract}

Keywords: crab-eating fox, ilium fracture, femur fracture, pubis fracture, ischium fracture, wildlife.

Descritores: graxaim-do-mato, fratura de ílio, fratura de fêmur, fratura de púbis, fratura de ísquio, vida selvagem.

${ }^{1}$ Programa de Pós-graduação em Ciências Veterinárias (PPGCV), Universidade Federal do Rio Grande do Sul (UFRGS), Porto Alegre, RS, Brazil. ${ }^{2}$ Núcleo de Conservação e Reabilitação de Animais Silvestre/PRESERVAS: Hospital de Clínicas Veterinárias (HCV)-UFRGS, Porto Alegre. CORRESPONDENCE: M.M. Alievi [marcelo.alievi@ufrgs.br - Tel.: +55 (51) 33088142]. Faculdade de Veterinária. Av. Bento Gonçalves n. 9090. Bairro Agronomia. CEP 91540-000 Porto Alegre, RS, Brazil. 


\section{INTRODUÇÃO}

O cachorro-do-mato (Cerdocyon thous) é uma espécie de canídeo silvestre que possui ampla ocorrência na América do Sul. Possui hábitos alimentares oportunistas e dieta onívora, o que leva à maior ocorrência destes animais em áreas próximas aos centros urbanos. Como consequência disto, acidentes por interação antrópica são comuns [6,9].

$\mathrm{O}$ atendimento desses animais, vítimas de atropelamentos, em centros de triagem, criadouros e zoológicos é frequente [6]. Fraturas, luxações e escoriações estão entre as lesões mais comuns nos traumas [3]. As técnicas de redução de fraturas para canídeos silvestres citadas na literatura são semelhantes às de cães domésticos [4,5], sendo encontrados poucos estudos para osteossínteses em Cerdocyon thous.

Fraturas da pelve representam de 20 a $30 \%$ das fraturas observadas em pequenos animais, enquanto as de fêmur representam de 20 a $25 \%$, sendo a maioria causada por acidentes automobilísticos [3,18]. Quando fraturados (pelve e fêmur), a biomecânica da deambulação fica comprometida, prejudicando a função motora do animal, sendo recomendada a estabilização cirúrgica para o desenvolvimento de condições estruturais adequadas para locomoção [7].

Partindo deste contexto, devido aos poucos estudos que descrevam técnicas de osteossíntese para cachorro-do-mato, o presente trabalho tem como objetivo relatar as osteossínteses realizadas em ílio e fêmur de um espécime de Cerdocyon thous com histórico de atropelamento, tendo em vista a aptidão do animal para retorno à vida livre.

\section{CASO}

Um espécime de cachorro-do-mato (Cerdocyon thous), fêmea, adulta, com 5,2 kg de massa corporal, proveniente de vida livre, encontrado próximo ao município de Cambará do Sul, no Parque Nacional de Aparados da Serra, foi encaminhado ao atendimento clínico com histórico de atropelamento. $\mathrm{O}$ atendimento emergencial foi prestado em clínica particular, sendo relatados sinais clínicos de choque e politraumatismo. Posteriormente a estabilização, o animal foi encaminhado ao atendimento hospitalar.

Na chegada, foi observado que o animal se encontrava em estado alerta de consciência, em decúbito esternal, sem sustentação do peso corporal sobre os membros pélvicos. Para realização dos exames clínicos e complementares, foi realizada contenção química com associação de cetamina ${ }^{1}\left(\right.$ Cetamin $^{\circledR} 5 \mathrm{mg} . \mathrm{kg}^{-1}$ ) e midazolam ${ }^{2}$ (Dormire ${ }^{\circledR} 0,5 \mathrm{mg} . \mathrm{kg}^{-1}$ ) por via intramuscular, sendo este protocolo utilizado em todos os manejos em que foram necessárias contenções químicas do animal durante a internação.

No exame físico geral, foram observadas escoriações cutâneas próximas à mandíbula e região tarsal, além de hematomas, edema e crepitações no membro pélvico direito. Não foram verificadas alterações para parâmetros vitais, como temperatura retal $\left(\mathrm{T}^{\circ} \mathrm{R}\right)$, frequência cardíaca (FC), frequência respiratória (FR), glicemia, pressão arterial sistólica (PAS), tempo de preenchimento capilar (TPC) e coloração de mucosas.

No exame ortopédico, foi constatado crepitação e instabilidade na região femoral direita, assim como assimetria, instabilidade e crepitação na região pélvica direita. Para uma melhor avaliação, o animal foi encaminhado ao exame radiográfico que demonstrou a presença de fratura completa transversa simples da diáfise do fêmur direito, fratura oblíqua do corpo do ílio direito, fratura transversa do púbis e fratura transversa do corpo do ísquio direito com deslocamento medial da porção cranial (Figura 1). Foi realizada ultrassonografia abdominal total, radiografia torácica e análise hematológica e bioquímica sérica (albumina, proteína plasmática total, alanina aminotransferase, ureia e creatinina) através de amostras de sangue coletadas da veia jugular, não sendo observadas alterações do padrão fisiológico da espécie nestes parâmetros [5].

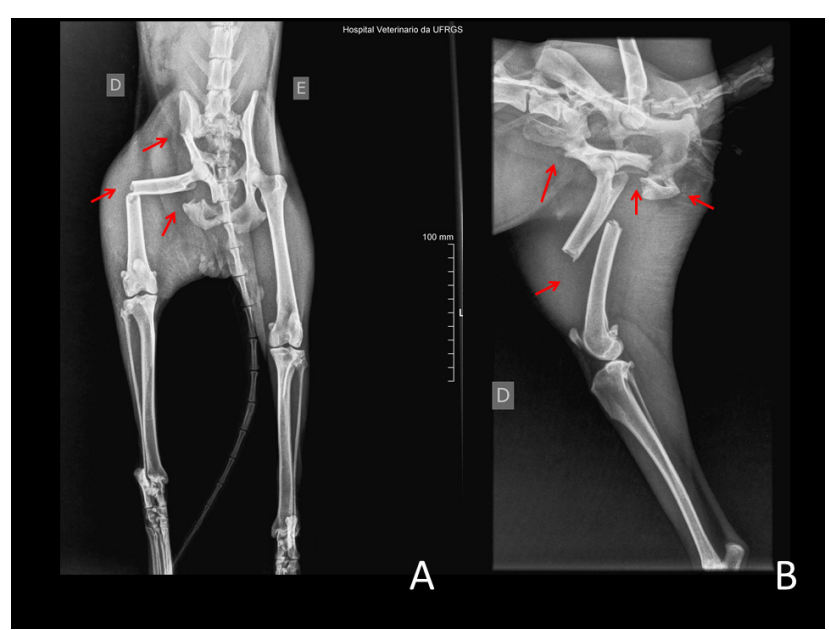

Figura 1. Radiografias ventrodorsal (A) e lateral (B) de pelve e fêmur direito em Cerdocyon thous. Observação de fratura completa transversa de diáfise de fêmur direito, fratura oblíqua de corpo de ílio direito, fratura transversa de púbis e fratura transversa de corpo de ísquio direito com deslocamento medial de porção cranial, indicado nas setas. [Fonte: Arquivo do HCV/UFRGS]. 
Como terapêutica, foi instituído: fluidoterapia com solução de ringer com lactato de sódio (Solução fisiológica de Ringer com lactato 3 , $40 \mathrm{~mL} \cdot \mathrm{kg}^{-1} / \mathrm{dia}, \mathrm{SC}$, 2 dias), enrofloxacino ${ }^{4}$ (Enrologic $₫ 5 \mathrm{mg} \mathrm{kg}^{-1}, \mathrm{VO}, \mathrm{q} 12 \mathrm{~h}$, 20 dias), meloxicam ${ }^{5}$ (Maxicam® $0,2 \mathrm{mg} \cdot \mathrm{kg}^{-1}, \mathrm{IM}, \mathrm{q} 24 \mathrm{~h}$, 3 dias) e cloridrato de $\operatorname{tramadol}^{6}$ (Tramal ${ }^{\circledR} 4 \mathrm{mg} \cdot \mathrm{kg}^{-1}, \mathrm{IM}$, q12 h, 10 dias). $\mathrm{O}$ animal foi encaminhado para realização das osteossínteses do ílio e fêmur 24 h após o atendimento.

As medicações pré-anestésicas utilizadas foram cetamina (10 mg. $\mathrm{kg}^{-1}$, IM), midazolam (0,4 mg. $\left.\mathrm{kg}^{-1}, \mathrm{IM}\right)$ e metadona ${ }^{2}$ (Mytedon ${ }^{\circledR} 0,4 \mathrm{mg} \cdot \mathrm{kg}^{-1}$, IM). Após tricotomia ampla da região pélvica, lombar e de todo membro pélvico direito, foi realizado venóclise na veia cefálica, mediante o uso de cateter $n^{\circ}$ $22 \mathrm{G}$, pelo qual administrado cefalotina ${ }^{7}$ (Cefalotina sódica $25 \mathrm{mg} \cdot \mathrm{kg}^{-1}$, IV, q 2h) como antibioticoprofilaxia e propofol $^{2}$ (Propovan ${ }^{\circledR} 5 \mathrm{mg} \cdot \mathrm{kg}^{-1}, \mathrm{IV}$ ) para indução anestésica. Em seguida foi realizada intubação com tubo endotraqueal $\left(n^{\circ} 6,0\right)$, e manutenção anestésica com isoflurano vaporizado em oxigênio a $100 \%$ em sistema avalvular sem reinalação de gases (Baraka) e fluidoterapia com solução de cloreto de sódio $0,9 \%$ (10 mL. $\mathrm{kg}^{-1}$ hora, IV). Posterior a antissepsia da região lombar com clorexidine $2 \%$, foi realizada bloqueio peridural com cloridrato de bupivacaína $0,5 \%$ sem vasoconstritor $^{2}$ (Neocaína ${ }^{\circledR} 1 \mathrm{mg} \cdot \mathrm{kg}^{-1}$ ) e sulfato de morfina ${ }^{2} 1 \%$ (Dimorf® $0,1 \mathrm{mg} \cdot \mathrm{kg}^{-1}$ ). Os parâmetros vitais foram verificados através de monitor multiparamétrico (frequência respiratória, pulso periférico, saturação de oxigênio, frequência cardíaca [ritmo e traçado eletrocardiográfico], temperatura esofágica e pressão arterial não invasiva), não sendo observada nenhuma intercorrência no transoperatório.

Com o paciente em decúbito lateral esquerdo e após a antissepsia e colocação dos campos cirúrgicos, foi realizado o acesso ao corpo do ílio conforme técnica descrita por Johnson [8] para cães. A redução da fratura foi realizada com auxílio de uma pinça óssea fixada sobre a borda dorsal do fragmento caudal do ílio, deslocando-o caudolateralmente até o alinhamento anatômico. A estabilização da fratura foi realizada mediante o uso de uma placa $2,7 \mathrm{~mm}$ bloqueada e 6 parafusos, sendo 4 bloqueados e 2 corticais.

A segunda incisão foi realizada para acesso ao foco de fratura do fêmur, através do acesso craniolateral da coxa conforme a técnica descrita por Johnson [8] para cães. Após a exposição, a fratura foi reduzida com o auxílio de pinças ósseas e estabilizada mediante o uso de uma placa $2,7 \mathrm{~mm}$ bloqueada e 6 parafusos ( 4 bloqueados e 2 corticais).
Após as estabilizações do ílio e do fêmur, foi realizada rafia dos planos musculares com sutura contínua simples, redução do subcutâneo com padrão de sutura contínua simples e dermorrafia com pontos isolados simples, mediante uso de mononáilon 3-0.

No pós-operatório imediato, foi realizada radiografia controle (Figura 2), na qual foi constatada coaptação adequada dos focos de fratura, porém na estabilização da pelve foi observado que um parafuso estava próximo a articulação coxofemoral, o que levou a reintervenção cirúrgica, para retirada deste. Além disto, foi observado moderado desvio medial em eixo longitudinal do ílio, com deslocamento medial do acetábulo.

Durante o período de 48 horas de pós-operatório, o animal ficou sob monitoração para avaliação de micção e defecação, onde foram observados normúria e normoquesia. Em aproximadamente cinco dias o animal já permanecia em estação e com deambulação claudicante. Após 14 dias foi realizada contenção química do animal para avaliação física e retirada de pontos, onde foi observada cicatrização adequada da ferida cirúrgica. Após 26 dias de tratamento o animal foi transferido para recinto externo de $10 \mathrm{~m}^{2}$.

Durante a internação foram realizadas avaliações clínicas periódicas que não demonstraram alterações sugestivas de complicações do quadro do paciente. $\mathrm{O}$ acompanhamento radiográfico foi realizado na $2^{\mathrm{a}}, 5^{\mathrm{a}}$ e $10^{\mathrm{a}}$ semana de pós-operatório (Figura 3). $\mathrm{Na}$ radiografia da $5^{\mathrm{a}}$ semana de pós-operatório foi observado o início da formação de calo ósseo e na da $10^{a}$ semana a consolidação e o remodelamento ósseo. Observou-se ainda redução do diâmetro do

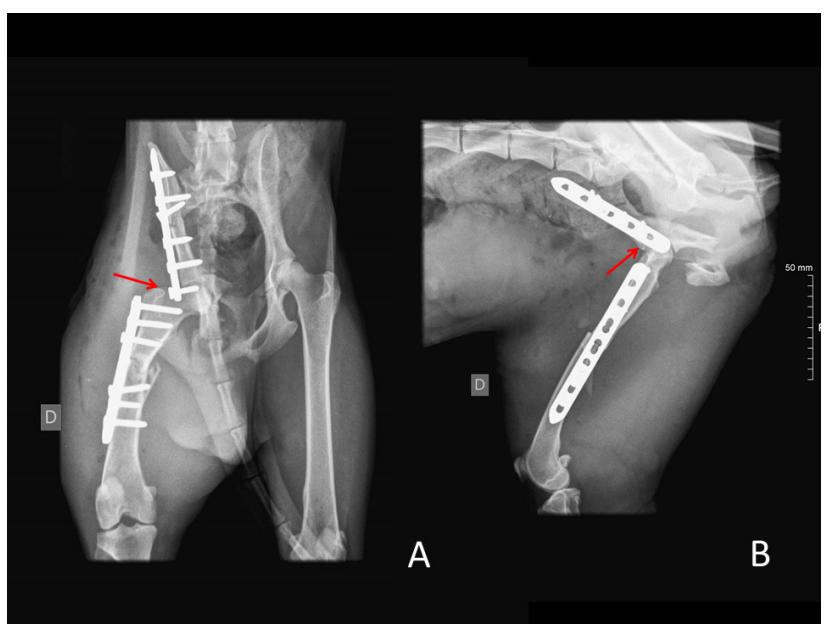

Figura 2. Radiografias ventrodorsal (A) e lateral (B) respectivamente, realizadas no pós- operatório imediato em Cerdocyon thous. Observação de coaptação adequada dos focos de fratura para corpo do ílio e diáfise de fêmur direito e observação de parafuso (seta) em pelve próximo a articulação coxofemoral. [Fonte: Arquivo do HCV/UFRGS]. 
canal pélvico e deslocamento medial do acetábulo. Clinicamente o animal deambulava adequadamente, defecava sem dificuldades e não apresentava sinais de domesticação.

Após 120 dias de tratamento, o animal foi submetido à implantação de microchip, por via subcutânea, na região interescapular e posteriormente foi encaminhado à destinação pelos órgãos ambientais. Aproximadamente 15 dias antes da soltura, foi realizado um novo manejo com contenção química, para colocação de radiocolar VHS na região cervical do animal.

A soltura em vida livre foi realizada 18 semanas após o procedimento cirúrgico. Após 30 e 60 dias da soltura, foram realizados monitoramentos por radiotelemetria no Parque, onde não foram observados sinais do rádio do animal.

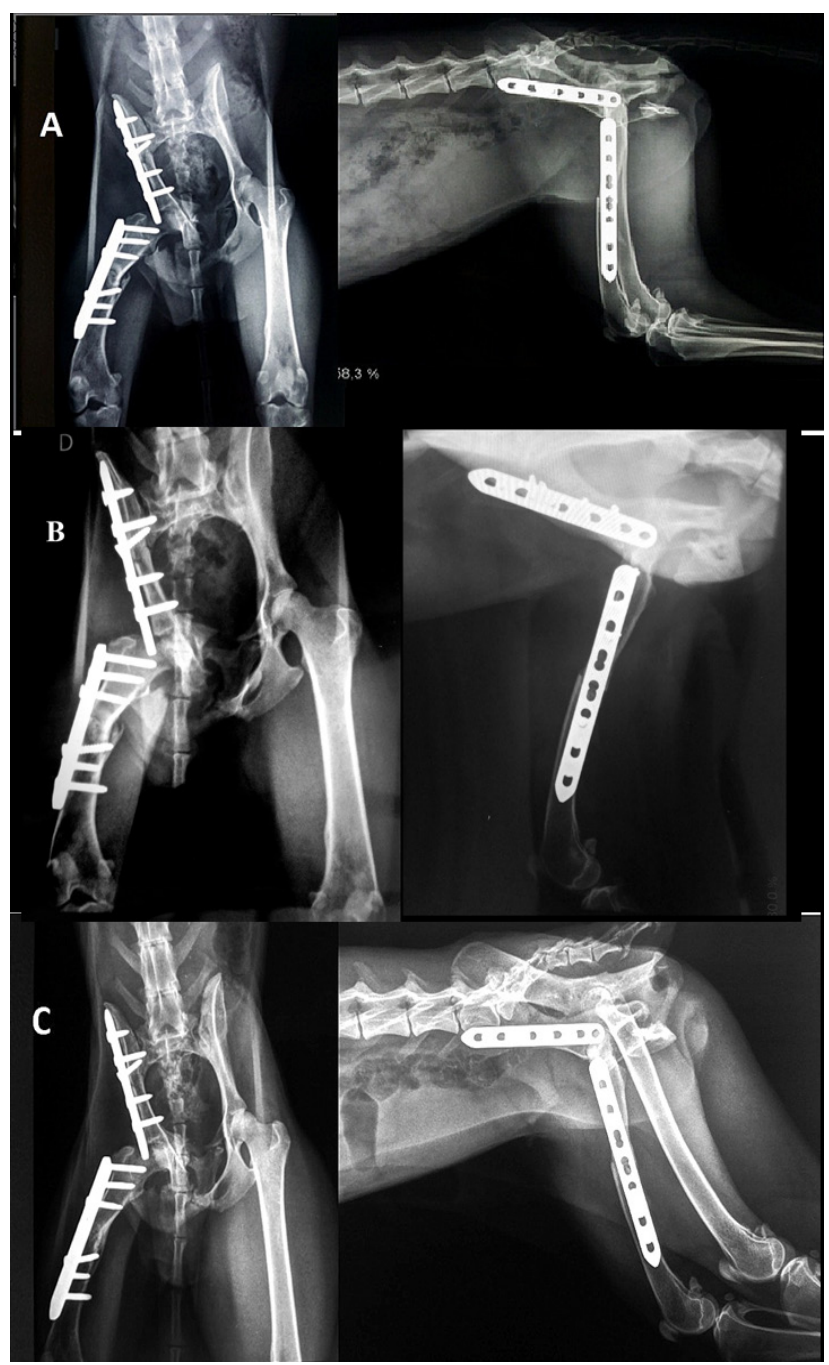

Figura 3. Radiografias ventrodorsal e lateral respectivamente, realizadas como controle pós-operatório na $2^{\mathrm{a}}(\mathrm{A}), 5^{\mathrm{a}}$ (B) e $10^{\mathrm{a}}$ (C) semana de pósoperatório de osteossíntese de ílio e fêmur direito em Cerdocyon thous, demonstrando evolução de cicatrização óssea após procedimento. [Fonte: Arquivo do HCV/UFRGS].

\section{DISCUSSÃO}

Para animais asselvajados, implantes ortopédicos internos (placa e parafusos) são os mais indicados para osteossíntese, visto que requerem mínima manipulação pós-operatória e, com isso, diminuem os riscos de complicações decorrentes deste manejo e o contato com as pessoas, permitindo a manutenção do comportamento asselvajado do animal mesmo em cativeiro $[4,16]$.

Para as fraturas de pelve, apesar da ampla descrição de implantes para correção de fraturas ilíacas, foi utilizada placa bloqueada, a qual apresenta como vantagens sobre as demais técnicas o fato de ser estável e não permitir movimentação entre o implante e o osso, conferindo estabilidade e rigidez ao sistema. Pelos parafusos serem bloqueados à placa, estes não exigem um perfeito alinhamento/coaptação com o osso e modelagem perfeita da placa em relação ao eixo ósseo a ser fixado [3,12], o que facilita a sua fixação ao corpo do ílio e ao fêmur e preserva o suprimento sanguíneo ósseo.

Dentre as complicações observadas no caso clínico, cita-se o uso de placa longa na osteossíntese do corpo do ílio e o estreitamento do canal pélvico como as mais relevantes. Com frequência, o ílio fraturado apresenta o segmento caudal deslocado craniomedialmente, reduzindo a amplitude do canal pélvico e, por vezes, comprometendo o nervo ciático que se localiza medialmente [14]. No que se refere ao estreitamento do canal pélvico e ao ciclo reprodutivo da espécie, os cachorros-do-mato apresentam comportamento monogâmico, de gestação de 3 a 6 filhotes por ninhada e aleitamento materno durante aproximadamente três meses. Devido às ninhadas serem relativamente grandes (número de indivíduos por parto), os filhotes são relativamente pequenos - com aproximadamente $120 \mathrm{~g}$ ao nascer [1]-, logo se espera que não ocorram complicações durante o parto.

Ao que tange o tratamento de fraturas pélvicas, a ênfase cirúrgica é dada para aquelas que envolvem a região sacroilíaca, ílio e acetábulo, por se tratar do eixo de transmissão das forças do membro pélvico ao esqueleto axial [16]. Isto corrobora a opção de tratamento conservador para púbis e ísquio, tendo em vista a possibilidade de redução da fratura de ísquio através da redução da fratura o ílio e o difícil acesso cirúrgico e riscos de intercorrências causando lesões de nervo ciático. O tratamento conservador é suficiente na maioria das fraturas do ramo isquiático e tuberosidade isquiática, 
no entanto, em alguns animais origina-se, com a fratura, um fragmento ósseo de elevado tamanho que é deslocado distalmente pela contração dos tendões musculares, o que provoca um desconforto significativo, fato não demonstrado no espécime em questão [3].

Para as fraturas de púbis, as indicações cirúrgicas não estão bem definidas na literatura, no entanto, a cirurgia está recomendada se existir instabilidade marcada, deslocamento acentuado, hérnia pré-púbica ou dor, assim como para as fraturas de ísquio [11]. No caso clínico descrito, não foi considerada a necessidade de intervenção cirúrgica, tendo em vista a inexistência dessas complicações e o efetivo controle da dor.

Para as fraturas de fêmur, segundo Brinker $e t$ al. [3], o uso de placas e parafusos no tratamento de fraturas transversais diafisárias é eficaz, obtendo-se prognóstico favorável para cães e gatos. Neste tipo de fratura, embora seja classificada como "simples", a ocorrência de não-uniões é comum, devido a subestimação das forças biomecânicas que atuam sobre o foco da fratura. As principais forças que atuam são a rotação e compressão, podendo haver sobrecarga de forças biomecânicas sobre os implantes metálicos comprometendo sua fixação. No presente caso clínico não foram identificadas essas complicações, visto que foram respeitados os princípios básicos de fixação de placas, sendo transfixadas 6 corticais em cada segmento fraturado (3 parafusos bicorticais), obtendo-se adequada consolidação óssea após 10 semanas. Para fraturas transversais, o uso de parafusos abrangendo 6 a 8 corticais é o suficiente para estabilizar a fratura, fornecendo fixação rígida e gerando condições para consolidação óssea adequada [3].

Apesar de terem sido encontrados poucos estudos ortopédicos para C. thous, a osteossíntese é recomendada em canídeos com múltiplas lesões músculo-esqueléticas e que possuem comportamento agressivo e/ou ativo [1,10]. Bettis [2] afirma que a estabilização rígida proporciona deambulação precoce e cicatrização adequada do osso, como observado no caso clínico.

A destinação para soltura em vida livre e o monitoramento por radiotelemetria foi uma decisão conjunta entre os órgãos ambientais do Estado e da União. A decisão foi baseada na manutenção do comportamento asselvajado sem evidências de mansidão do espécime, a ausência de restrições sa- nitárias, bem como a biologia populacional e o fluxo genético visto que a soltura do animal foi realizada próximo ao local de resgate [17].

As técnicas de radiotelemetria buscam, em geral, elucidar padrões de movimentação, territorialidade e utilização de recursos, bem como avaliar parâmetros de sobrevivência e dispersão de uma determinada espécie [19]. A translocação e reintrodução de carnívoros constituem estratégias que requerem várias considerações, sejam elas, genéticas, demográficas, comportamentais, epidemiológicas ou mesmo de requerimentos de habitats particulares de cada espécie, sendo estas levadas em consideração para soltura do espécime [13]. Por isso optou-se pela soltura do animal sob monitoramento por radiotelemetria, e desde a sua soltura até o presente momento foram realizadas duas saídas de campo, onde não foram captados sinais de frequência de rádio (VHS). Tal fato se deve possivelmente ao relevo acidentado do local [20] e a formação de barreiras físicas que impedem a passagem das ondas, corroborando a importância da continuidade do monitoramento e estudos que envolvam a soltura pós-reabilitação de animais silvestres.

Conclui-se que os métodos utilizados para osteossínteses de ílio e fêmur, associados ao manejo de pré e pós-operatório foram satisfatórios para reabilitação de espécime de Cerdocyon thous, culminando com a aptidão para soltura do animal à vida livre. Todavia, existe a necessidade de continuidade do monitoramento para poder se inferir o sucesso da reabilitação do espécime em questão.

\section{MANUFACTURERS}

${ }^{1}$ Syntec Brasil Indústria Farmacêutica. Santana da Parnaíba, SP, Brazil

${ }^{2}$ Cristália Produtos Químicos Farmacêuticos Ltda. Itapira, SP, Brazil. ${ }^{3}$ Eurofarma Laboratórios S.A. Itapevi, SP, Brazil.

${ }^{4}$ Pharmalogic Animal Health. Monami. Jacareí, SP, Brazil.

${ }^{5}$ Ouro Fino Saúde Animal. Cravinhos, SP, Brazil.

${ }^{6}$ Laboratórios Pfizer. Itapevi, SP, Brazil.

${ }^{7}$ Aurobindo Pharma. Anápolis, GO, Brazil.

Acknowledgements. Agradecemos a Secretaria do Meio Ambiente e Desenvolvimento Sustentável (SEMA-RS), a Fundação Zoobotânica (FZB) pelo apoio na translocação e destinação do animal, ao Instituto Chico Mendes (ICMBio) e a BIOTA pelo material concedido para monitoramento do animal.

Declaration of interest. The authors report no conflicts of interest. The authors alone are responsible for the content and writing of the paper. 


\section{REFERENCES}

1 Bettis C.W. 1998. Fraturas pélvicas. In: Slatter D. (Ed). Manual de cirurgia de pequenos animais. 3.ed. São Paulo: Manole, pp.2094-2113.

2 Berta A. Cerdocyon thous (Carnivora: Canidae). Mammalian Species. 186: 1-4.

3 Brinker W.O., Piermattei D.L. \& Flo G.L. 2009. Fraturas e condições ortopédicas do membro pélvico. In: Ortopedia e Tratamento de Fraturas de Pequenos Animais. 4.ed. Barueri: Manole, pp.491-814.

4 Ferrigno C.R.A., Schmaedecke A. \& Ferraz V. 2014. Ortopedia. In: Cubas Z.S., Silva J. C. R. \& Catão-Dias J.L. (Eds). Tratado de animais selvagens: medicina veterinária. 2.ed. São Paulo: Roca, pp.2095-2112.

5 Gomes M.S. 2007. Carnivora - Canidae (Lobo-guará, cachorro-do-mato, raposa-do-campo). In: Cubas Z.S., Silva J.C.R. \& Catão-Dias J.L. (Eds). Tratado de animais selvagens: medicina veterinária. São Paulo: Roca, pp.492-504.

6 Gonçalves J.A., Almeida V.L., Ribeiro D.G.A., Gomes Sobrinho R.F., Milliole R.R., Pires J.R. \& Soares M.A. 2016. Mamíferos silvestres resgatados na zona oeste do rio de Janeiro - RJ. Novo Enfoque: Caderno Saúde e Meio Ambiente. 21: 8-13.

7 Hulse D. \& Hyman B. 2007. Biologia e biomecânica das fraturas. In: Slatter D. (Ed). Manual de cirurgia de pequenos animais. 3.ed. São Paulo: Manole, pp.1785-1792.

8 Johnson A.L. 2008. Fundamentos da cirurgia ortopédica e manejo de fraturas. In: Fossum T.W. (Ed). Cirurgia de pequenos animais. 3.ed. Rio de Janeiro: Elsevier, pp.930-1014.

9 Kasper C.B., Trinca C.S., Sanfelice D., Mazim F.D. \& Trigo T.C. 2014. Os carnívoros. In: Gonçalves G., Quintela F.M. \& Freitas T.R.O. (Eds). Mamíferos do Rio Grande do Sul. Porto Alegre: Pacartes, pp.162-190.

10 Kawamoto F.Y.K., Perles L., Ribeiro J.M., Werther K., Minto B.W., Franco G.G., Rossignoli P.P. \& Simamura A.C.A.A. 2016. Tratamento cirúrgico de fratura de pelve e disjunção sacro-ilíaca em cachorro-do-mato (Cerdocyon thous). In: Anais do XII Congresso de Cirurgia do CBCAV e II Congresso Internacional do CBCAV (Águas de Lindóia, Brasil). p.134.

11 Kemper B., Gonçalves L.P., Vieira M.O., Figueiredo M.L., Severo M.S. \& Tudury E.A. 2011. Consequências do trauma pélvico em cães. Ciência Animal Brasileira. 12(2): 311-321.

12 Leal L.M., da Silva G.T., Marinho P.V.T., Prada T.C., Minto B.W. \& Machado M.R.F. 2014. Osteosynthesis of the iliac body with locking plate and stabilization of sacroiliac luxation with locking screw - case report. Medvep: Revista Científica de Medicina Veterinária - Pequenos Animais e Animais de Estimação. 12(40): 218-223.

13 Miller B., Ralls K., Reading R.P., Scott M.J. \& Estes J. 1999. Biological and technical considerations of carnivore translocation: a review. Animal Conservation. 2: 59-68.

14 Olmstead M.L. 1995. Fractures of the bone of the hind limbs. In: Olmstead M.L. (Ed). Small animal orthopedics. St. Louis: Mosby, pp.219-228.

15 Orsini H. \& Bondan E.F. 2014. Fisiopatologia do Estresse. In: Cubas Z.S., Silva J.C.R. \& Catão-Dias J.L. (Eds). Tratado de animais selvagens: medicina veterinária. 2.ed. São Paulo: Roca, pp.35-45.

16 Payne J.T. 1993. Selecting a method for managing pelvic fractures in dogs and cats. The Veterinary Record. 88(10): 969-973.

17 Rio Grande do Sul. 2015. Lei Complementar 140 de 2011/ Portaria Secretária do Ambiente e Desenvolvimento Sustentável (SEMA-RS), 177, de 30 de novembro de 2015. [Fonte:http://www.famurs.com.br/arq_upload/20160226124601_ Portaria\%20SEMA\%20n\%C2\%BA\%20177_.pdf. [Accessed online in January 2017].

18 Roehsig C., Rocha L.B., Barauna Junior D., Chioratto R., Silva S.R.A.M., Kemper B., Araújo F.P., Almeida A.C.M. \& Tuduri E.A. 2008. Fixação de fraturas ilíacas em cães com parafusos, fios de aço e cimento ósseo de polimetilmetacrilato. Ciência Rural. 38(6): 1675-1681.

19Soisalo M. \& Cavalcanti S. 2006. Estimating the density of a jaguar population in the Brazilian Pantanal using camera traps and capture-recapture sampling in combinations with GPS radio-telemetry. Biological Conservation. 129: 487-496.

20 Wildner W. 2004. Excursão Virtual aos Aparados da Serra - RS/SC: aspectos geológicos e turísticos - Cânions do Itaimbezinho e Fortaleza. Porto Alegre: CPRM, 88p. 